\title{
Diabetic Gastroparesis: A Forgotten Disease
}

\section{Patrizio Tatti*}

Endocrinology and Diabetes Unit, Italy

Gastroparesis is a rather frequent condition in which the motility of the stomach is extremely delayed or absent, and is usually consequential to many other seemingly unrelated diseases, although rarely idiopathic forms do exist. The disorder is rather common in type 1 diabetes, with a figure of about $10 \%$ and is most prevalent in conditions of poor blood glucose control and in developing countries, although this has not been definitely proven [1]. Recently a private company announced that "Gastric emptying of solids is delayed in 30\% to 50\% of patients with diabetes mellitus, functional dyspepsia and gastroesophageal reflux disease [2]. Despite high prevalence of symptoms, the rate of gastroparesis diagnosis is low among those with type 2 diabetes" Such prevalence was estimated at $14 \%$ of type 1 diabetics in the U.S $(165,000)$ and 2.1 million type 2 diabetics $(9.4 \%)$. These figures should be considered in the light of the companys' interest in the launch of a new specific product for gastroparesis. However the study is led by acknowledged authorities in the field, rigorous and based on a rigorous and validated methodology, the disease may mimic many other disorders, or maybe masqueraded by other afflictions [3]. The diagnosis is difficult and requires a high index of suspicion and a sophisticated dynamic study. The most common symptoms in the advanced chronic forms are $[4,5]$ :

\section{Nausea}

\section{Vomiting}

Early satiety

\section{Regurgitation}

Fullness, bloating

The best understood presentation in the one that appears in diabetes, although accounting for only $30 \%$ of cases [6]. Unfortunately, the condition is difficult to diagnose, as the symptoms are subtle, and common to many other disorders. Gastroparesis is frequently associated with GI reflux that overshadows the diagnosis, while in fact the reflux the result of the overfilled un-emptied stomach. In its most advanced form Gastroparesis is a debilitating illness disrupting the life of the patient with unremitting vomiting, apparently independent of meals [7]. When the gastroparesis appears in a diabetic the resulting blood glucose profile is absolutely irregular with peaks and valleys that may appear at any time of the day without apparent explanation [8]. This phenomenon as a matter of fact maybe a clue to the diagnosis. In these patients the use of insulin or any insulin secretagogues is particularly hazardous because of the unpredictable blood glucose levels that make timing of the insulin administration impossible, and may even lead to near-lethal blood glucose level falls. On the other hand the use of GLP1 analogues is equally dangerous because these agents cause further inhibition of the gastrointestinal motility [4]. With the more widespread use of these agents a $\mathrm{n}$ increase in the cases of gastroparesis should be expected. The patients should be questioned accurately for sign and symptoms of the disease before anyone of these classes of drugs is used. Apparently the same does not hold true for the DPP-IV inhibitors [9]. Sometimes the patients are so accustomed to vomiting that they neglect to inform their physician. Many patients with gastroparesis paradoxically do not lose weight, but do present a consistent degree of malnutrition with cutaneous and hematologic manifestations. Because diabetic neuropathy is a systemic disease, associated manifestation, e.g. cardiovascular neuropathy or disruptions of the circadian rhythms are often seen in such patients. The disappearance of the nocturnal dipping of blood pressure is an example of a commonly associated symptom of diabetic gastroparesis.

Understanding fully the pathophysiology of the disease is difficult, as is its treatment. Although many pharmacologic treatments have been proposed, from antibiotics to pro/motility agents, one of the most effective is the surgical insertion of a neuro regulatory "impulse generator", which apparently works on the "nerves of Cajal "[10] Unfortunately in many patients these devices only offer partial relief and the quality of life remains poor. The prognosis for long-term survival is not favourable, probably because of the associated cardiovascular autonomic neuropathy. A further problem appears when the disease is effectively treated because after the end of an "inferno" to which they had become accustomed, recovering a normal life poses many hurdles. Some stick to their use of ingesting a huge amount of calories, which now they do not waste with vomit, and gain a lot of weight. Some may even require psychiatric help. There is no definitive proof that the disease can be prevented in diabetes with an intensive blood glucose control, although may seem plausible.

In summary Gastroparesis is a common and underdiagnosed disease that deserves more attention in earlier diagnosis and treatment. There is urgent need for papers that can help to gain insight into pathophysiology of the disturbed motility to lead to a better cure. We hope that the pages of the Journal will host additional data to help "see through the glass".

\section{References}

1. Kofod-Andersen K, Tarnow L (2012) Prevalence of gastroparesis-related symptoms in an unselected cohort of patients with Type 1 diabetes. J Diabetes Complications 26: 89-93.

2. Horowitz M, Su YC, Rayner CK, Jones KL (2001) Gastroparesis: prevalence, clinical significance and treatment. Can J Gastroenterol 15: 805-813.

3. http://www.reuters.com/article/2013/05/20/ma-rhythmepidemiology-idUSnPNC L16880+1e0+PRN20130520

4. Camilleri M, Parkman HP, Shafi MA, Abell TL, Gerson L; American College of Gastroenterology (2013) Clinical guideline: management of gastroparesis. Am J Gastroenterol 108: 18-37.

5. Hornbuckle K, Barnett JL (2000) The diagnosis and work-up of the patient with gastroparesis. J Clin Gastroenterol 30: 117-124

*Corresponding author: Patrizio Tatti M.D., Endocrinology and Diabetes Unit, ASL Roma H, Ospedale S. Giuseppe, Rome, Italy, Tel: + (39) 069327 3094; Email: info@patriziotatti.it

Received July 22, 2014; Accepted July 24, 2014; Published August 01, 2014

Citation: Tatti P (2014) Diabetic Gastroparesis: A Forgotten Disease. J Diabetes Metab 5: e116 doi:10.4172/2155-6156.1000e116

Copyright: (c) 2014 Tatti P. This is an open-access article distributed under the terms of the Creative Commons Attribution License, which permits unrestricted use, distribution, and reproduction in any medium, provided the original author and source are credited. 
6. Buckles DC, McCallum RW (2004) Treatment of Gastroparesis. Curr Treat Options Gastroenterol 7: 139-147.

7. Farup CE, Leidy NK, Murray M, Williams GR, Helbers L, et al. (1998) Effect of domperidone on the health-related quality of life of patients with symptoms of diabetic gastroparesis. Diabetes Care 21: 1699-1706.

8. Merio R, Festa A, Bergmann H, Eder T, Eibl N, et al. (1997) Slow gastric emptying in type I diabetes: relation to autonomic and peripheral neuropathy, blood glucose, and glycemic control. Diabetes Care 20: 419-423.

9. Vella A, Bock G, Giesler PD, Burton DB, Serra DB, et al. (2007) Effects of dipeptidyl peptidase-4 inhibition on gastrointestinal function, meal appearance and glucose metabolism in type 2 diabetes. Diabetes 56: 1475-1480.

10. Patrizio T, Felice S, Annabel B (2011) Diabetic Gastrointestinal Neuropathy: Elusive Diagnosis and Difficult Treatment. The Open Clinical Trials Journal 3: 20-25. 